\title{
Yersinia Pseudotuberculosis Modulates Regulatory T Cell Stability via Injection of Yersinia Outer Proteins in a Type III Secretion System-Dependent Manner
}

\author{
Ahmed Elfiky ${ }^{1}$, Agnes Bonifacius ${ }^{1}$, Joern Pezoldt ${ }^{1}$, Maria Pasztoi ${ }^{1}$, Paweena Chaoprasid ${ }^{2}$, Pooja Sadana ${ }^{3}$, \\ Nagla El-Sherbeeny ${ }^{4}$, Magda Hagras ${ }^{4}$, Andrea Scrima ${ }^{3}$, Petra Dersch ${ }^{2}$ and Jochen Huehn ${ }^{\text {* }}$ \\ ${ }^{1}$ Experimental Immunology, Helmholtz Centre for Infection Research, Braunschweig, Germany \\ ${ }^{2}$ Molecular Infection Biology, Helmholtz Centre for Infection Research, Braunschweig, Germany \\ ${ }^{3}$ Structural Biology of Autophagy, Helmholtz Centre for Infection Research, Braunschweig, Germany \\ ${ }^{4}$ Department of Clinical Pharmacology, College of Medicine, Suez Canal University, Ismailia, Egypt
}

Received: 21 June 2018; accepted: 13 July 2018

\begin{abstract}
Adaptive immunity is essentially required to control acute infection with enteropathogenic Yersinia pseudotuberculosis (Yptb). We have recently demonstrated that Yptb can directly modulate naïve $\mathrm{CD}^{+} \mathrm{T}$ cell differentiation. However, whether fully differentiated forkhead box protein P3 $\left(\right.$ Foxp $\left.^{+}\right)$regulatory $\mathrm{T}$ cells (Tregs), fundamental key players to maintain immune homeostasis, are targeted by Yptb remains elusive. Here, we demonstrate that within the $\mathrm{CD}^{+} \mathrm{T}$ cell compartment Yptb preferentially targets Tregs and injects Yersinia outer proteins (Yops) in a process that depends on the type III secretion system and invasins. Remarkably, Yop-translocation into ex vivo isolated Foxp $3^{+}$Tregs resulted in a substantial downregulation of Foxp3 expression and a decreased capacity to express the immunosuppressive cytokine interleukin-10 (IL-10). Together, these findings highlight that invasins are critically required to mediate Yptb attachment to Foxp $3^{+}$Tregs, which allows efficient Yop-translocation and finally enables the modulation of the Foxp $3^{+}$Tregs' suppressive phenotype.
\end{abstract}

Keywords: regulatory T cells, Yersinia pseudotuberculosis, Yersinia outer proteins, invasins, cytotoxic necrotizing factor y, Foxp3

\section{Introduction}

Regulatory T cells (Tregs) are central players to confer tolerance towards self and harmless foreign antigens. They are characterized by expression of the lineage-specification transcription factor forkhead box protein P3 (Foxp3), which not only is a reliable marker to identify Tregs, but also is a key transcriptional regulator for the suppressive phenotype of these immunoregulatory cells [1]. Foxp $3^{+}$Tregs are integral to mediate immune tolerance. Thus, manipulation of Foxp3 expression by either enhancing its stability in the context of autoimmune diseases or ameliorating its stability in the context of cancer or chronic infections poses a promising strategy to further improve therapeutic approaches targeting Foxp3 ${ }^{+}$ Tregs [2, 3].

In general, most infections overcoming epithelial barrier functions result in an inflammatory environment in the underlying tissues and the draining lymph nodes (LN), driving the establishment of $\mathrm{T}$ helper cell 1 (Th1) and Th17 responses. Importantly, the infection-induced skewing towards effector $\mathrm{T}$ cell responses can result in limitation of interleukin-2 (IL-2), a cytokine critically required to maintain stability of Foxp3 expression in Tregs [4-8]. In the context of gastrointestinal infections, we could recently show that upon oral infection with Yersinia pseudotuberculosis (Yptb), naïve $\mathrm{CD}^{+} \mathrm{T}$ cells get directly targeted by the pathogen, which ultimately drives naïve $\mathrm{CD}^{+} \mathrm{T}$ cell differentiation towards Th17 cells rather than

*Author for correspondence: Inhoffenstrasse 7, 38124 Braunschweig, Germany; E-mail: jochen.huehn@helmholtz-hzi.de; Phone: +49 5316181 3310; Fax: +4953161813399.
Tregs [9]. Yet, the extent to which the Treg population is targeted by Yptb is unknown.

Yptb can efficiently translocate effector molecules, so called Yersinia outer proteins (Yops), into target cells [10]. Yops activate and abrogate homeostatic localization of Ras homologousGTPase (Rho-GTPase) proteins, mitigate phosphorylation patterns, and limit activity of different small G-proteins, resulting in the deregulation of actin cytoskeleton assembly and celltype dependently altering molecular functionalities [11]. The cytotoxic necrotizing factor $y$ (CNFy) is a Yptbsecreted protein that exhibits its immunomodulatory effect by activating the Rho-GTPase proteins Rho A, Rac1, and Cdc42 [12-14]. Rho-GTPase proteins are key components of $\mathrm{T}$ cell development, activation, differentiation, and migration, acting via modulation of the $T$ cell cytoskeleton [15]. In particular, activation of Rap1, a Rho-GTPase protein, was shown to enhance both thymic and peripheral Treg development [16]. Importantly, effective translocation of Yops via the type III secretion system (T3SS) is only possible via direct cell-Yptb contact, established by different invasins (inv). Particularly, invasin $A$ acts as a potent $\beta_{1}$ integrin ligand (mainly via $\alpha_{4} \beta_{1}$ integrin) [17-19], potentially allowing effective binding to $\beta_{1}$ integrin-expressing cells, including epithelial cells and Foxp $3^{+}$ Tregs [20].

Although it is known that Yptb can modulate naïve $\mathrm{CD}^{+} \mathrm{T}$ cell differentiation relying on the T3SS [9], direct modulation of Foxp $3^{+}$Treg stability by Yptb virulence factors has not been described, yet. Here, we demonstrated that Yptb preferentially targets Tregs across the $\mathrm{T}$ cell compartment in a T3SS-dependent manner and directly modulates the stability and functional properties of Tregs by decreasing Foxp3 and IL-10 expression, respectively.

This is an open-access article distributed under the terms of the Creative Commons Attribution-NonCommercial 4.0 International License (https://creativecommons.org/licenses/by-nc/4.0/), which permits unrestricted use, distribution, and reproduction in any medium for non-commercial purposes, provided the original author and source are credited, a link to the CC License is provided, and changes - if any - are indicated. 


\section{Materials and Methods}

Mouse Strains. Foxp $3^{\mathrm{hCD} 2}$ reporter mice $(\mathrm{C} 57 \mathrm{BL} / 6$ background) were used as organ donors for the isolation of $\mathrm{T}$ cells within the study. All animals were bred and housed under specific pathogen-free conditions at the Helmholtz Centre for Infection Research (Braunschweig, Germany).

Bacterial Strains and Recombinant Proteins. $Y$. pseudotuberculosis strains, namely Yptb-WT-Bla, Yptb$\Delta$ T3SS-Bla, Yptb- $\Delta$ inv and Yptb- $\Delta$ CNFy-Bla, were used throughout the study [13]. Overnight cultures of Yptb strains were grown at $25{ }^{\circ} \mathrm{C}$ in Lysogeny broth (LB) medium, and subsequently diluted at 1:50, followed by $2 \mathrm{~h}$ of culture at $25{ }^{\circ} \mathrm{C}$ and $3 \mathrm{~h}$ at $37{ }^{\circ} \mathrm{C}$. Cultures were performed in the presence of $50 \mu \mathrm{g} / \mathrm{mL}$ kanamycin (Sigma Aldrich) for all strains except Yptb- $\Delta$ CNFy-Bla, which was cultured in the presence of $50 \mu \mathrm{g} / \mathrm{mL}$ chloramphenicol (Sigma Aldrich). Recombinant invasins $\mathrm{A}, \mathrm{D}$, and $\mathrm{E}$ as well as CNFy were produced as described before [13, 21, 22].

Antibodies and Flow Cytometry. Fluorochromeconjugated anti-human CD2 (RPA-2.10), anti-CD4 (RM4-5), anti-CD8 $\alpha$ (53-6.7), anti-CD11c (N418), anti-CD45R (RA36B2), anti-CD62L (MEL-14), anti-F4/80 (BM8), anti-Foxp3 (FJK-16S), anti-IFN $\gamma$ (XMG1.2), anti-IL-10 (JES5-16E3), and anti-IL-17A (eBio17B7) antibodies were purchased from BioLegend and eBioscience. Prior to cytokine staining, cells were stimulated with phorbol 12-myristate 13-acetate (PMA, $10 \mathrm{ng} / \mathrm{mL})$ and Ionomycin $(0.5 \mu \mathrm{g} / \mathrm{mL})$ for $4 \mathrm{~h}$, with Brefeldin A $(10 \mu \mathrm{g} / \mathrm{mL})$ added for the final two h (all Sigma-Aldrich). Surface staining was performed for $15 \mathrm{~min}$ on ice in PBS (Gibco) containing $0.2 \%$ bovine serum albumin (BSA, Sigma-Aldrich). Intracellular Foxp3 staining was performed using Foxp3 staining buffer set (eBioscience) according to the manufacturer's instructions. Absolute cell numbers were determined using Accurri C6 Cytometer (Becton Dickinson). Dead cell exclusion was carried out using LIVE/DEAD Fixable Blue Dead Cell Stain (Thermo Fisher Scientific). Cells were washed, resuspended in phosphate buffered saline/ bovine serum albumin (PBS/BSA), and measured at LSR-II SORP with Diva software 6.1 (BD Biosciences). Data were analyzed using FlowJo software (Tree Star).

In Vitro Treg Stability Assay. Total $\mathrm{CD}^{+} \mathrm{T}$ cells were enriched from spleens and LNs of Foxp $3^{\text {hCD2 }}$ mice via magnetic sorting using autoMACS (Miltenyi Biotec). From these CD4-enriched cells, CD4 $4^{+} \mathrm{Foxp}^{\mathrm{hCD} 2+}$ Tregs or $\mathrm{CD} 4^{+}$Foxp $3^{\mathrm{hCD} 2-}$ conventional $\mathrm{T}$ cells (Tconv) were sorted on Aria II SORP (BD Biosciences) or MoFlo XDP (Beckman Coulter) cell sorters. Tregs or Tconv were co-cultured with Yptb-WT-Bla, Yptb- $\Delta$ T3SS-Bla, Yptb- $\Delta$ inv, or Yptb- $\Delta$ CNFyBla at the indicated multiplicity of infection (MOI) at $37{ }^{\circ} \mathrm{C}$ for $1 \mathrm{~h}$ and washed twice with Roswell Park Memorial Institute (RPMI) medium supplemented with $50 \mu \mathrm{g} / \mathrm{mL}$ gentamicin (Sigma-Aldrich) to eliminate bacteria. Cells were labeled with the proliferation dye CellTrace ${ }^{\mathrm{TM}}$ Violet (CTV, Thermo Fisher Scientific) and cultured in 96-well plates pre-coated with antiCD3 (17A2, $1 \mu \mathrm{g} / \mathrm{mL})$ and anti-CD28 (37.51, $1 \mu \mathrm{g} / \mathrm{mL})$ antibodies (both BioLegend) in complete RPMI (cRPMI, 10\% FCS, $50 \mathrm{U} / \mathrm{mL}$ penicillin, $50 \mathrm{U} / \mathrm{mL}$ streptomycin, $1 \mathrm{mM}$ sodium pyruvate, $25 \mathrm{mM}$ HEPES, and $50 \mu \mathrm{M} \beta$ mercaptoethanol, all Gibco) supplemented with $50 \mathrm{ng} / \mathrm{mL}$ IL-2 (R\&D) and $50 \mu \mathrm{g} / \mathrm{mL}$ gentamicin (Sigma-Aldrich) for 3 days. For studies involving recombinant proteins (CNFy, invasin A, $\mathrm{D}$, and E), Tregs were cultured as described above in the presence of the indicated proteins. At the end of the culture, cells were harvested and analyzed by flow cytometry.

$\beta$-Lactamase Reporter Assay. For in vitro analysis of Yop translocation, total $\mathrm{CD}^{+} \mathrm{T}$ cells were isolated from spleens and LNs of Foxp $3^{\text {hCD2 }}$ mice using anti-CD4-Microbeads and the autoMACS Pro separation system (Miltenyi Biotec). Subsequently, cells were co-cultured with Yptb-WT-Bla or Yptb- $\Delta$ T3SS-Bla at the indicated MOIs at $37{ }^{\circ} \mathrm{C}$ for $1 \mathrm{~h}$ and washed twice with RPMI medium supplemented with $50 \mu \mathrm{g} / \mathrm{mL}$ gentamicin to eliminate bacteria. Cells were stained for cell surface markers anti-CD4 and anti-hCD2 and labeled with CCF4-AM, using the LiveBLAzer-FRET B/G loading kit (Thermo Fisher Scientific) for $1 \mathrm{~h}$ at $24{ }^{\circ} \mathrm{C}$ in the presence of $1.5 \mathrm{mM}$ probenecid (Sigma-Aldrich) and $50 \mu \mathrm{g} / \mathrm{mL}$ gentamicin. Finally, cells were harvested and analyzed by flow cytometry.

Yptb Binding to Tregs. Tregs were isolated as described above and co-cultured with Yptb-WT-Bla or Yptb- $\Delta$ inv at a MOI of 50 for $1 \mathrm{~h}$ at $37{ }^{\circ} \mathrm{C}$. Subsequently, cell-Yptb single cell solutions were repeatedly washed by centrifugation at $400 \mathrm{~g}$ to sediment eukaryotic cells, limiting the sedimentation of prokaryotic Yptb. Cell-Yptb single cell solutions were resuspended in LB medium, and serial dilutions were plated on LB plates. These were kept at $37^{\circ} \mathrm{C}$ for $36 \mathrm{~h}$, and colony forming units (CFU) per Treg were calculated.

Statistical Analysis. For all figures, each data point represents the mean of at least 3 technical replicates per independent experiment, if not stated otherwise. For comparison of unmatched groups, two-tailed Mann-Whitney statistical test was applied. The comparison of more than 2 groups was performed by Kruskall-Wallis test followed by Dunn's Multiple Comparison test. All data are presented as mean or mean $\pm \mathrm{SD}$, and $p$-values $<0.05$ are considered as significant $(* p<0.05 ; * * p<0.01 ; * * * p<0.001 ; * * * * p<$ 0.0001). Prism software (GraphPad) was applied for all statistical analyses and graphs.

Ethics. Animals were handled with appropriate care and welfare in accordance with good animal practice as defined by FELASA and the national animal welfare body GV-SOLAS under supervision of the institutional animal welfare officer, and all efforts were made to minimize suffering.

\section{Results}

Yptb Preferentially Targets Tregs Within the $\mathrm{CD}^{+} \mathrm{T}$ Cell Compartment. We have recently shown that Yptb can directly interact with $\mathrm{CD}^{+}{ }^{+} \mathrm{T}$ cells and efficiently translocate Yops into both Tconv and Foxp3 ${ }^{+}$Tregs in vivo [9]. Interestingly, this translocation allowed a direct modulation of naïve $\mathrm{CD}^{+}{ }^{+} \mathrm{T}$ cell differentiation and resulted in an enhanced Th17 differentiation and decreased induction of Foxp $3^{+}$Tregs in vitro [9]. Here, we further dissected the efficiency of Yptb to target Tconv and Foxp3 ${ }^{+}$Tregs in vitro. To this end, we used the $\beta$-lactamase reporter assay [13], which relies on the chromosomal integration of a YopE- $\beta$-lactamase fusion protein (Yptb-WT-Bla) and allows the identification of cells into which Yops were successfully translocated by Yptb. Total $\mathrm{CD} 4^{+} \mathrm{T}$ cells, obtained from Foxp $3^{\mathrm{hCD} 2}$ reporter mice, were co-cultured with Yptb-WT-Bla at a MOI of 35, followed by labeling with the dye CCF4-AM (green fluorescence), which turns into blue fluorescence upon conversion by $\beta$-lactamase. Flow cytometric analysis of Foxp $3^{\text {hCD2- }}$ Tconv and Foxp $3^{\text {hCD2 } 2+}$ Tregs revealed a significantly higher frequency of blue cells among Tregs when compared to Tconv (Figure 1). These in vitro data, which are in line with our previous observations in vivo [9], demonstrate that Yptb preferentially targets Tregs within the $\mathrm{CD} 4^{+} \mathrm{T}$ cell compartment.

Yptb Suppresses Foxp3 and IL-10 Expression in Tregs in a T3SS-Dependent Manner. Based on the preferential targeting of Foxp3 $3^{+}$Tregs and our previous observation that Yptb can directly modulate naïve $\mathrm{CD} 4^{+} \mathrm{T}$ cell differentiation 
a

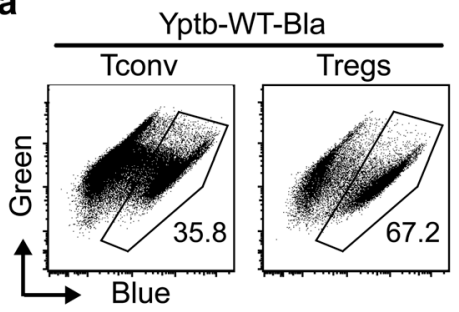

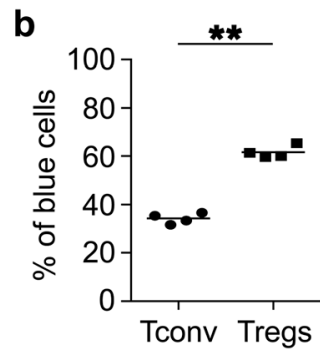

Figure 1. Tregs are efficiently targeted by Yptb for translocation of effector proteins. CD4 ${ }^{+} \mathrm{T}$ cells isolated from Foxp $3^{\mathrm{hCD} 2}$ reporter mice were co-cultured with Yptb-WT-Bla at $37^{\circ} \mathrm{C}$ for $1 \mathrm{~h}$ at MOI 35, labelled with CCF4-AM and subsequently analyzed by flow cytometry. Alive unmodulated cells are labelled in "Green", and cells that were modulated by Yptb show "Blue" fluorescence. (a) Representative dot plots show frequency of translocation in the indicated T-cell subsets. Numbers indicate the frequency of modulated "Blue" cells in the corresponding gates. (b) Scatterplot summarizes the frequency of modulated "Blue" cells among the indicated T-cell subsets. Data were pooled from 4 independent experiments $(* * p<0.01 ;$ Bla, $\beta$-lactamase; MOI, multiplicity of infection)

[9], we next asked if Yptb can also modulate the phenotype of already differentiated Foxp $3^{+}$Tregs. Thus, sorted Foxp $3^{\text {hCD2+ }}$ Tregs were co-cultured with Yptb-WT for $1 \mathrm{~h}$, and subsequently, Yptb function was abrogated by applying gentamicin to the culture. Co-cultures with Yptb- $\Delta$ T3SS served as a control to investigate the importance of Yop translocation [9]. Afterwards, the modulated Foxp $3^{\mathrm{hCD} 2+}$ Tregs were cultured for 3 days in vitro on plate-bound anti$\mathrm{CD} 3 / 28$ in the presence of high-dose IL-2. At the end of the culture, flow cytometric analysis revealed a significantly decreased Foxp3 expression in Tregs that were previously modulated by Yptb-WT, when compared to unmodulated Tregs, as indicated by both a reduced frequency of Foxp3 $3^{+}$ cells, as well as a lower geometric mean fluorescence intensity (gMFI) (Figure 2a and b). Interestingly, this reduction of Foxp3 expression was fully dependent on Yop translocation as Tregs that were modulated by Yptb- $\Delta$ T3SS did show neither a reduced frequency of Foxp $3^{+}$cells nor a lowered gMFI. It has to be mentioned that Tregs modulated with Yptb-WT, but not with Yptb- $\Delta \mathrm{T} 3 \mathrm{SS}$, showed an increased cell death and

a

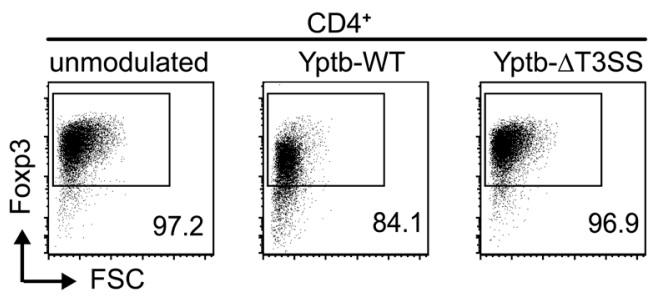

b
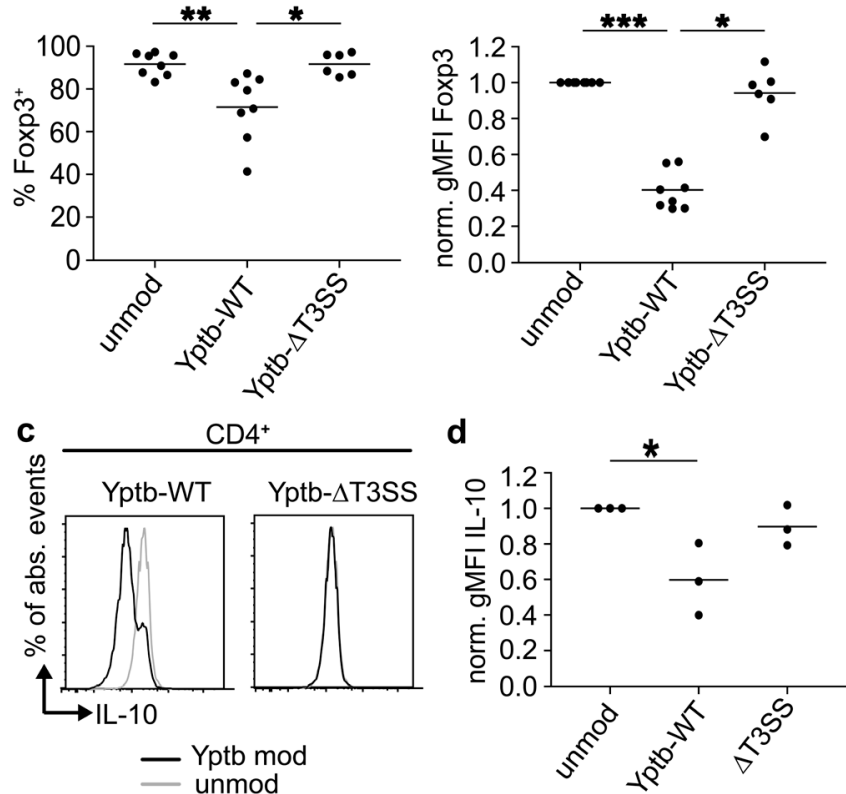

Figure 2. Foxp3 and IL-10 expression are suppressed in Tregs by Yptb in a T3SS-dependent manner. Foxp $3^{+}$Tregs isolated from Foxp $3^{\text {hCD2 }}$ reporter mice were co-cultured with Yptb-WT-Bla or Yptb- $\Delta$ T3SS-Bla at $37{ }^{\circ} \mathrm{C}$ for $1 \mathrm{~h}$ at MOI 50 . Subsequently, bacteria were killed by antibiotics treatment, and Tregs were cultured on anti-CD3/CD28-coated plates in the presence of IL-2. At day 3 of culture, cells were analyzed by flow cytometry. (a) Representative dot plots show Foxp3 expression among alive $\mathrm{CD} 4^{+}$cells from indicated cultures. Numbers indicate the frequency of Foxp $3^{+}$cells in the corresponding gates. (b) Scatterplots summarize the frequencies of Foxp $3^{+}$cells (left) and gMFI of Foxp3 (normalized to unmodulated control, right) among alive $\mathrm{CD}^{+}$cells from the indicated cultures. (c) Representative histograms show IL-10 expression among alive $\mathrm{CD} 4^{+}$cells from the indicated cultures. (d) Scatterplot summarizes gMFI of IL-10 among alive CD4 ${ }^{+}$cells from indicated cultures. Each dot represents mean of 3 technical replicates. Data were pooled from 3 to 8 independent experiments $\left({ }^{*} p<0.05 ; * * p<0.01 ; * * * p<0.001\right.$; gMFI, geometric mean fluorescence intensity; MOI, multiplicity of infection; mod, modulated; unmod, unmodulated; T3SS, Type III secretion system) 
impaired proliferative response at day 3 of the culture when compared to unmodulated controls (Supplementary Figure 1), most likely due to the direct interference with $\mathrm{T}$ cell receptor downstream signaling as previously reported [9]. However, the abovementioned reduction of Foxp3 expression upon modulation with Yptb-WT cannot be due to a selective killing of Tregs and survival of Foxp $3^{-}$cells as Foxp $3^{\text {hCD2+ }}$ Tregs sorted to highest purity were used in these in vitro cultures. In accordance with the reduced Foxp3 expression, we also observed a significantly decreased IL-10 expression when Tregs were modulated by Yptb-WT, while again no effect was observed upon modulation by Yptb- $\Delta$ T3SS (Figure $2 \mathrm{c}$ and $\mathrm{d}$ ). Importantly, the reduced Foxp3 and IL-10 expression was not accompanied by an increase in IL-17A, IFN $\gamma$, T-bet and ROR $\gamma \mathrm{t}$ expression (data not shown), suggesting that the modulated Tregs do not acquire an inflammatory phenotype upon downregulation of Foxp3 expression.

Viewed as a whole, our data demonstrate that Yptb can directly affect Foxp3 and IL-10 expression within Tregs, a process that fully relies on the T3SS, indicating that the injection of Yops functions as a key factor in modulating the stability and functional properties of Tregs.

Invasins and CNFy are Key Effector Virulence Factors for Effective Yop Translocation into Tregs. Translocation of
Yops via the T3SS can be enhanced by invasins and CNFy $[13,18]$. To determine whether these effector molecules are required for the reduction of Foxp3 expression, sorted Foxp $3^{\text {hCD2+ }}$ Tregs were co-cultured with Yptb either lacking all invasins (Yptb- $\triangle$ inv) or CNFy (Yptb- $\triangle \mathrm{CNFy}$ ) for $1 \mathrm{~h}$, followed by an elimination of all remaining Yptb by gentamicin treatment. Subsequently, the modulated Foxp $3^{\mathrm{hCD} 2+}$ Tregs were cultured for 3 days in vitro on platebound anti-CD3/28 in the presence of high-dose IL-2. Remarkably, at the end of the culture, neither Tregs modulated by Yptb- $\Delta$ inv nor Yptb- $\Delta$ CNFy showed any decrease in Foxp3 expression when compared to Tregs modulated by Yptb-WT (Figure 3a and b), suggesting that invasins and CNFy are both critically required for the reduction of Foxp3 expression. As abovementioned, Tregs modulated with YptbWT, but neither with Yptb- $\Delta$ inv nor with Yptb- $\Delta C N F y$, showed an increased cell death and impaired proliferative response at day 3 of the culture when compared to unmodulated controls (Supplementary Figure 1). Yet, in vitro culture of sorted Foxp $3^{\mathrm{hCD} 2+}$ Tregs in the presence of recombinant $\mathrm{CNFy}$, invasin $\mathrm{A}$, invasin $\mathrm{D}$, or invasion $\mathrm{E}$ did not result in any reduced Foxp3 expression (Figure 3c). Together, these findings implicate that CNFy and invasins cannot directly affect Foxp3 expression within Tregs on their

a

$\mathrm{CD}^{+}$
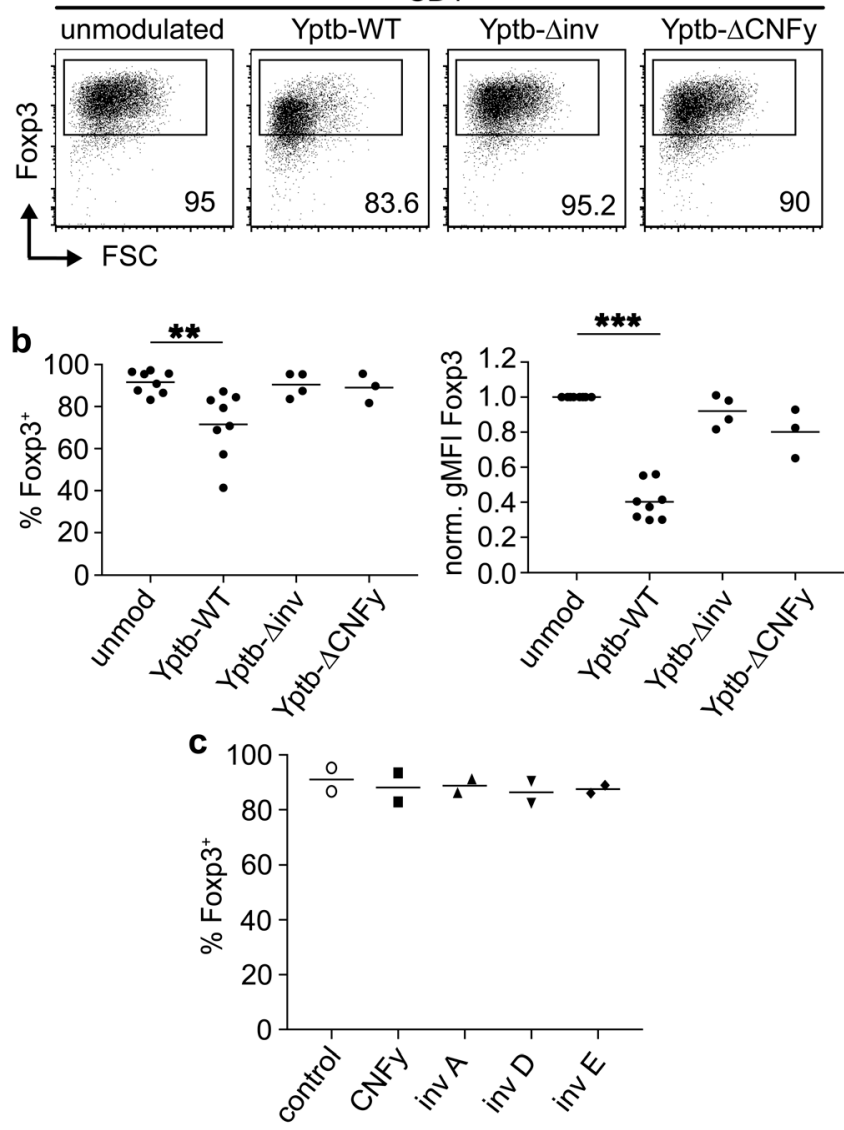

Yptb recombinant proteins

Figure 3. Invasins and CNFy are required for efficient Yop translocation into Tregs. Foxp $3^{+}$Tregs isolated from Foxp ${ }^{\text {hCD2}}$ reporter mice were co-cultured with Yptb-WT, Yptb- $\Delta$ inv or Yptb- $\Delta$ CNFy at $37{ }^{\circ} \mathrm{C}$ for $1 \mathrm{~h}$ at MOI 50 . Subsequently, bacteria were killed by antibiotics treatment, and Tregs were cultured on anti-CD3/CD28-coated plates in the presence of IL-2. At day 3 of culture, cells were analyzed by flow cytometry. (a) Representative dot plots show Foxp3 expression among alive $\mathrm{CD}^{+}$cells from the indicated cultures. Numbers indicate the frequency of Foxp $3^{+}$ cells in the corresponding gates. (b) Scatterplots summarize frequencies of Foxp3 ${ }^{+}$cells (left) and gMFI of Foxp3 (normalized to unmodulated control, right) among alive $\mathrm{CD} 4^{+}$cells from the indicated cultures. Unmodulated controls and Tregs modulated with Yptb-WT, same as in Figure 2b. (c) Foxp $3^{+}$Tregs isolated from Foxp $3^{\text {hCD2}}$ reporter mice were cultured as described above in the presence of CNFy, invasin A, D, or E. At day 3 of culture, cells were analyzed by flow cytometry. Scatterplot summarizes the frequencies of Foxp $3^{+}$cells among alive CD4 ${ }^{+}$cells from the indicated cultures. Each dot represents mean of 3 technical replicates. Data were pooled from 2 to 8 independent experiments $\left({ }^{* *} p<0.01 ; * * * p<\right.$ 0.001. inv, invasin; CNFy, cytotoxic necrotizing factor y; unmod, unmodulated; MOI, multiplicity of infection) 


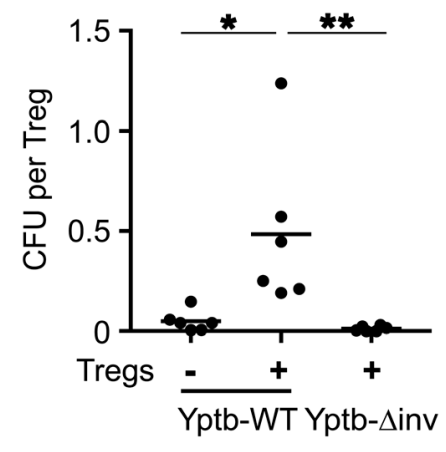

Figure 4. Attachment of Yptb to Tregs essentially requires invasins. Foxp $3^{+}$Tregs isolated from Foxp $3^{\mathrm{hCD} 2}$ reporter mice were co-cultured with Yptb-WT or Yptb- $\Delta$ inv at $37^{\circ} \mathrm{C}$ for $1 \mathrm{~h}$ at MOI 50 . Yptb-WT without Tregs served as control. After repeated washing and centrifugation to remove unbound bacteria, the remaining cell pellet was plated in serial dilutions, and CFU per Treg were determined. "+" indicates the presence of Tregs in the co-cultures. Each dot represents a biological unicate. Data were pooled from 6 independent experiments $\left({ }^{*} p<0.05 ; * * p<0.01\right.$; inv, invasin; MOI, multiplicity of infection; $\mathrm{CFU}$, colony forming unit)

own, yet they are both independently required for efficient translocation of Yops via the T3SS and thereby contribute to the modulation of the Tregs' phenotype.

Invasins are Required for Attachment of Yptb to Tregs. Having demonstrated that invasins are critically required for the reduction of Foxp3 expression in Tregs upon modulation by Yptb, we next aimed to determine the molecular mechanism involved and asked if invasins can foster the attachment of Yptb to Tregs. To this end, sorted Foxp $3^{\text {hCD2+ }}$ Tregs were cocultured with Yptb-WT or Yptb- $\Delta$ inv for $1 \mathrm{~h}$. Subsequently, unbound bacteria were removed by repeated washing and centrifugation, and the remaining cell pellet was plated in serial dilutions to determine the number of bacteria bound to the cells. Strikingly, while Tregs co-cultured with Yptb-WT showed a high number of attached Yptb when compared to the negative control (Yptb-WT without Tregs), any specific binding was observed when Tregs were co-cultured with Yptb- $\Delta$ inv (Figure 4). These findings indicate that invasins are crucially required to mediate Yptb attachment to Tregs, thereby enabling their efficient modulation through the translocation of Yops.

\section{Discussion}

Within the intestinal tract, effector and regulatory immune cell subsets need to be tightly balanced to maintain immune homeostasis and, at the same time, combat infections effectively. We have recently shown that Yptb directly interacts with $\mathrm{CD}^{+} \mathrm{T}$ cells, which results in an efficient translocation of Yops and a skewing of naïve $\mathrm{CD}^{+} \mathrm{T}$ cell differentiation from immunosuppressive Tregs to inflammatory Th17 cells [9]. Here, we expanded our findings on fully differentiated Tregs and could demonstrate that Yptb directly impairs Treg stability by suppressing Foxp3 expression and limiting their capacity to produce IL-10.

During acute infections the inflammatory environment limits Treg expansion and their immunosuppressive functions to enable the establishment of effector and memory $\mathrm{T}$ cell responses and subsequently an efficient eradication of the invading pathogens $[18,23,24]$. Therefore, pathogens including Mycobacteria, Salmonella, Helicobacter pylori, and helminths have evolved mechanisms to utilize the suppressive function of Tregs in order to support initial infection or establish chronicity [25]. In contrast to these pathogens, we here could show that Yptb directly interacts with Tregs and impairs lineage stability by interfering with proper Foxp3 expression, in line with our previous observation of Yptb-induced, ameliorated de novo Treg induction [9]. Together, these findings indicate that Yptb does not rely on Treg-mediated suppression of the immune response for successful establishment within the host.

Several molecular mechanisms have been identified that allow pathogens to directly modulate the function of Tregs [26], including the induction of Tregs by secretory antigens of Heligmosomoides polygyrus or tolerogenic polarization of dendritic cells by Helicobacter pylori $[23,27]$. Due to the effectiveness of pathogens to utilize Treg functionality, the molecular mechanism of pathogen-mediated modulation of Tregs is of high interest to develop novel therapeutic tools specifically targeting Tregs, e.g. in the context of tumor therapy [28]. Using various Yptb mutant strains lacking CNFy, invasins, or T3SS, we could demonstrate that Yop translocation via the T3SS is essential to inhibit Foxp3 expression. Importantly, CNFy-dependent inhibition of Foxp3 expression only took place in the presence of viable Yptb and not in the presence of recombinant CNFy, underlining the importance of the Yop translocation-enhancing properties of CNFy in this context [13]. Furthermore, Yptb requires close proximity to Tregs and utilizes invasin-mediated binding, presumably by interaction of invasin A with $\beta_{1}$ integrin [17-19], which is expressed at high levels on Foxp3 ${ }^{+}$Tregs [20]. Both, CNFy and invasins, were independently required for efficient translocation of Yops via the T3SS and thereby contributed to the modulation of the Tregs' phenotype. The extent to which the different Yops alter Treg function and mitigate Foxp3 expression requires further studies. Potentially interesting candidates might include YopH, a tyrosine phosphatase that was reported to impair proximal $\mathrm{T}$ cell receptor signaling by interfering with tyrosine-phosphorylation of downstream adapter molecules LAT and SLP-76 upon T cell activation [29, 30]. Additionally, YopJ can act as a deubiquitinating enzyme, interacting with stimulator of interferon genes (STING) that in turn inhibits IRF3 signaling [31], which might influence T cell differentiation. In the context of utilizing Yop translocation by combining different Yop functionalities, including the capacity of YopM to efficiently self-translocate [32], Yptb-originated effector proteins could be used to effectively target Tregs in the context of therapeutic approaches encompassing engineered bacteria for tumor therapy. So far, bacterial agents have been predominantly studied in tumor therapies using attenuated bacterial strains ranging from Clostridium novyi to auxotrophic Salmonella conferring effective anti-tumor immune responses with promising efficiency $[33,34]$. Thus, the capacity of Yptb to preferentially target Tregs in a T3SS-dependent manner and to directly modulate the stability and functional properties of Tregs by decreasing Foxp 3 and IL-10 expression, respectively, could be utilized in the future to leverage the development of novel Treg targeting approaches to boost antitumor immune responses.

\section{Funding Sources}

This work was supported by the Deutsche Forschungsgemeinschaft (SFB854 project B16 to JH). Work in the group of AS is partially funded by the Helmholtz Association (Young Investigator grant number VH-NG-727). PS gratefully acknowledges support by the President's Initiative and Networking Funds of the Helmholtz Association of German Research Centers (HGF) under contract number VH-GS-202.

\section{Authors' Contribution}

A.E., A.B., J.P., M.P., P.C., and P.S. performed experiments and interpreted data. N.E. and M.H. provided expertise and 
feedback. A.S. and P.D. provided reagents. A.E., A.B., J.P., M.P., P.D. and J.H. designed research, interpreted data, and wrote the manuscript.

\section{Conflict of Interest}

The authors declared no conflict of interest.

Acknowledgement. We thank Maria Ebel and Beate Pietzsch for technical assistance, Lothar Groebe and Maria Hoexter for cell sorting and Juhao Yang and Jana Niemz for valuable discussions.

\section{References}

1 Huehn J, Beyer M. Epigenetic and transcriptional control of Foxp3 regulatory T cells. Semin Immunol. 2015;27:10-8.

2 Miyara M, Ito Y, Sakaguchi S. T-cell therapies for autoimmune rheumatic diseases. Nat Rev Rheumatol. 2014.

3 Tanaka A, Sakaguchi S. Regulatory T cells in cancer immunotherapy. Cell Res. 2017;27:109-18.

4 Oldenhove G, Bouladoux N, Wohlfert EA, Hall JA, Chou D, Dos Santos L, et al. Decrease of Foxp $3^{+}$Treg cell number and acquisition of effector cell phenotype during lethal infection. Immunity. 2009;31:772-86.

5 Sakaguchi S, Vignali DA, Rudensky AY, Niec RE, Waldmann H. The plasticity and stability of regulatory T cells. Nat Rev Immunol. 2013;13:461-7.

6 Bhela S, Varanasi SK, Jaggi U, Sloan SS, Rajasagi NK, Rouse BT. The Plasticity and Stability of Regulatory $T$ Cells during Viral-Induced Inflammatory Lesions. J Immunol. 2017;199:1342-52.

7 Zhang Z, Zhang W, Guo J, Gu Q, Zhu X, Zhou X. Activation and Functional Specialization of Regulatory $\mathrm{T}$ Cells Lead to the Generation of Foxp3 Instability. J Immunol. 2017;198:2612-25.

8 Toomer KH, Malek TR. Cytokine Signaling in the Development and Homeostasis of Regulatory T cells. Cold Spring Harb Perspect Biol. 2018;10.

9 Pasztoi M, Bonifacius A, Pezoldt J, Kulkarni D, Niemz J, Yang J, et al. Yersinia pseudotuberculosis supports Th17 differentiation and limits de novo regulatory $\mathrm{T}$ cell induction by directly interfering with $\mathrm{T}$ cell receptor signaling. Cell Mol Life Sci. 2017;74:2839-50.

10 Viboud GI, Bliska JB. Yersinia outer proteins: role in modulation of host cell signaling responses and pathogenesis. Annu Rev Microbiol. 2005:59:69-89.

11 Zhang L, Mei M, Yu C, Shen W, Ma L, He J, Yi L. The Functions of Effector Proteins in Yersinia Virulence. Pol J Microbiol. 2016;65:5-12.

12 Hoffmann C, Pop M, Leemhuis J, Schirmer J, Aktories K, Schmidt G. The Yersinia pseudotuberculosis cytotoxic necrotizing factor (CNFY) selectively activates RhoA. J Biol Chem. 2004;279:16026-32.

13 Schweer J, Kulkarni D, Kochut A, Pezoldt J, Pisano F, Pils MC, et al The Cytotoxic Necrotizing Factor of Yersinia pseudotuberculosis (CNFY) Enhances Inflammation and Yop Delivery during Infection by Activation of Rho GTPases. PLoS Pathog. 2013;9:e1003746.

14 Wolters M, Boyle EC, Lardong K, Trulzsch K, Steffen A, Rottner K, et al. Cytotoxic necrotizing factor-Y boosts Yersinia effector translocation by activating Rac protein. J Biol Chem. 2013;288:23543-53.

15 Saoudi A, Kassem S, Dejean A, Gaud G. Rho-GTPases as key regulators of T lymphocyte biology. Small GTPases. 2014;5.

$16 \mathrm{Li} \mathrm{L}$, Kim J, Boussiotis VA. Rap1A regulates generation of T regulatory cells via LFA-1-dependent and LFA-1-independent mechanisms. Cell Immunol. 2010;266:7-13.
17 Arencibia I, Suarez NC, Wolf-Watz H, Sundqvist KG. Yersinia invasin, a bacterial beta1-integrin ligand, is a potent inducer of lymphocyte motility and migration to collagen type IV and fibronectin. J Immunol. 1997:159:1853-9.

18 Maldonado-Arocho FJ, Green C, Fisher ML, Paczosa MK, Mecsas J. Adhesins and host serum factors drive Yop translocation by yersinia into professional phagocytes during animal infection. PLoS Pathog. 2013;9: e1003415.

19 Deuschle E, Keller B, Siegfried A, Manncke B, Spaeth T, Koberle M, et al. Role of betal integrins and bacterial adhesins for Yop injection into leukocytes in Yersinia enterocolitica systemic mouse infection. Int J Med Microbiol. 2016;306:77-88.

20 Huehn J, Siegmund K, Lehmann JC, Siewert C, Haubold U, Feuerer $\mathrm{M}$, et al. Developmental stage, phenotype, and migration distinguish naiveand effector/memory-like CD $4^{+}$regulatory T cells. J Exp Med. 2004;199, 303-13.

21 Sadana P, Monnich M, Unverzagt C, Scrima A. Structure of the Y. pseudotuberculosis adhesin InvasinE. Protein Sci. 2017;26:1182-95.

22 Sadana P, Geyer R, Pezoldt J, Helmsing S, Huehn J, Hust M, et al. The invasin D protein from Yersinia pseudotuberculosis selectively binds the Fab region of host antibodies and affects colonization of the intestine. J Biol Chem. 2018;293:8672-90

23 Grainger JR, Smith KA, Hewitson JP, McSorley HJ, Harcus Y, Filbey $\mathrm{KJ}$, et al. Helminth secretions induce de novo $\mathrm{T}$ cell Foxp3 expression and regulatory function through the TGF-beta pathway. J Exp Med. 2010;207:2331-41.

24 Shafiani S, Dinh C, Ertelt JM, Moguche AO, Siddiqui I, Smigiel KS, et al. Pathogen-specific Treg cells expand early during mycobacterium tuberculosis infection but are later eliminated in response to Interleukin-12. Immunity. 2013;38:1261-70.

25 Boer MC, Joosten SA, Ottenhoff TH. Regulatory T-Cells at the Interface between Human Host and Pathogens in Infectious Diseases and Vaccination. Front Immunol. 2015;6, 217.

26 Garib FY, Rizopulu AP. T-Regulatory Cells as Part of Strategy of Immune Evasion by Pathogens. Biochemistry (Mosc). 2015;80:957-71.

27 Kao JY, Zhang M, Miller MJ, Mills JC, Wang B, Liu M, et al. Helicobacter pylori immune escape is mediated by dendritic cell-induced Treg skewing and Th17 suppression in mice. Gastroenterology. 2010;138:1046-54

28 Klages K, Mayer CT, Lahl K, Loddenkemper C, Teng MW, Ngiow SF, et al. Selective depletion of Foxp $3^{+}$regulatory $\mathrm{T}$ cells improves effective therapeutic vaccination against established melanoma. Cancer Res. 2010;70, 7788-99.

29 Yao T, Mecsas J, Healy JI, Falkow S, Chien Y. Suppression of T and B lymphocyte activation by a Yersinia pseudotuberculosis virulence factor, yopH. J Exp Med. 1999; 190:1343-50.

30 Gerke C, Falkow S, Chien YH. The adaptor molecules LAT and SLP-76 are specifically targeted by Yersinia to inhibit T cell activation. J Exp Med. 2005;201:361-71.

31 Cao Y, Guan K, He X, Wei C, Zheng Z, Zhang Y, et al. Yersinia YopJ negatively regulates IRF3-mediated antibacterial response through disruption of STING-mediated cytosolic DNA signaling. Biochim Biophys Acta. 2016;1863:3148-59.

32 Ruter C, Buss C, Scharnert J, Heusipp G, Schmidt MA. A newly identified bacterial cell-penetrating peptide that reduces the transcription of pro-inflammatory cytokines. J Cell Sci. 2010;123:2190-8.

33 Roberts NJ, Zhang L, Janku F, Collins A, Bai RY, Staedtke V, et al. Intratumoral injection of Clostridium novyi-NT spores induces antitumor responses. Sci Transl Med. 2014;6:249ra111.

34 Feloner S, Kocijancic D, Frahm M, Heise U, Rohde M, Zimmermann $\mathrm{K}$, et al. Engineered Salmonella enterica serovar Typhimurium overcomes limitations of anti-bacterial immunity in bacteria-mediated tumor therapy. Oncoimmunology. 2018;7:e1382791. 